\title{
Mesoscale Variations in the Heliospheric Magnetic Field and their Consequences in the Outer Heliosphere
}

\author{
L. A. Fisk \\ Department of Atmospheric, Oceanic, and Space Sciences, \\ University of Michigan, Ann Arbor, MI 48109-2143, USA
}

\begin{abstract}
This paper considers several different aspects of the magnetic field in the outer heliosphere and their consequences. It is noted first that many features of the heliospheric magnetic field are set back at the Sun, and that these processes result in variations in the heliospheric field on several distinct spatial scales. An equation is discussed that describes the interactions of mesoscale variations in the magnetic field with small-scale turbulence, and it is argued that this interaction can account for the creation of the observed superthermal tails on the particle distributions, and alter the expected behavior of the magnetic field at the termination shock.
\end{abstract}

\section{INTRODUCTION}

Currently, there are several interesting challenges in heliospheric physics. There is extensive evidence for large-scale acceleration of energetic particles in the solar wind. Gloeckler et al. [1,2], in data from both Ulysses and ACE, observe ubiquitous superthermal tails on the distributions of solar wind and interstellar pickup ions. These accelerated particles occur in the absence of shocks, suggesting that there is acceleration, perhaps by a statistical mechanism, in the ambient solar wind. The identification of the dominant mechanism remains unclear, although several viable theories have been proposed [e.g., 3,4,5,6]. There is also the important debate as to whether Voyager 1 has crossed the termination shock of the solar wind, with Krimigis et al. [7] arguing from low-energy energetic particle data that it has, and Burlaga et al. [8] from magnetic field data that it has not.

In this paper, we consider mesoscale variations in the heliosphere magnetic field, and argue that the dissipation of such variations by small-scale turbulence may account for the observed suprathermal tails accelerated in the solar wind and for altering the expected behavior of the magnetic field at the termination shock. In the limited space available for this paper, certain calculations and conclusions are summarized. More detailed discussions can be found in Fisk $[9,10]$. 


\section{MULTI-SCALE VARIATIONS OF THE HELIOSPHERIC MAGNETIC FIELD}

The origins of mesoscale variations in the heliospheric magnetic field are discussed by Zurbuchen et al. [11]. We summarize a few of the dominant processes here.

The principal process that influences the configuration of the heliospheric magnetic field is of course the rotation of the Sun. The rotation causes the magnetic field to develop a strong azimuthal component as the field expands radially outward with the solar wind. This results in the Parker spiral field configuration [12]. The azimuthal component falls off as one over the heliocentric radial distance, and thus is the dominant component in the outer heliosphere.

However, superimposed on the rotational motions of the Sun are other motions. The magnetic field of the Sun that forms the heliospheric field - the so-called open magnetic flux - is not stationary at the Sun. Such a large-scale motion should occur in the polar coronal holes at solar minimum, as was pointed out by Fisk [13]. The Sun differentially rotates, the poles rotating some $20 \%$ slower than the equator. The polar coronal holes, however, appear to rigidly rotate. The open magnetic field from the polar holes over expands from the coronal base. Considering these processes together, Fisk [13] concluded that in the frame of reference rotating at the equatorial rotation rate, the open magnetic field executes large systematic motions in latitude and longitude before being carried outward into the heliosphere.

In addition to these systematic motions, we have to introduce diffusive motions. The systematic motions driven by differential rotation must in some circumstances be balanced by diffusive transport $[14,15]$. The concept that magnetic fields diffuse at the Sun was introduced by Leighton [16], who argued that magnetic fields diffuse in random convective motions at the boundaries of supergranules. Such diffusion is an integral process in modern models for the evolution of the solar magnetic field, such as the models proposed by Wang et al. [17,18] or by Schrijver et al. [19,20]. Here we need a somewhat faster diffusion, which can be provided by the reconnection with loops whose sizes can exceed that of supergranules.

In fact, we should also expect that reconnection with loops, and diffusion by random convection at supergranules, occur everywhere on the Sun in varying degrees [15]. There are loops everywhere on the Sun - large ones outside of coronal holes, but smaller ones within coronal holes [21]. The diffusion process should create a braiding of the magnetic field on a moderately large scale - the size of loops, which, when projected out into the heliosphere, can at Earth be several hundreds up to a tenth of an AU in dimension.

All of these motions have other consequences as well. The solar wind is fast from coronal holes but slower from the open flux outside of coronal holes. There are motions across the interface between the two regions, and a resulting component of the magnetic field across the interface. For example, in the heliosphere at solar minimum there is high-speed wind at high latitudes, low-speed at low latitudes, and a shear plane in between. A component of the magnetic field across the shear plane is then stretched radially and in the outer heliosphere will be much more radial than predicted by the Parker spiral. The magnetic field in this model can go in and out of regions with 
different solar wind speeds and become stretched, and substantial local deviations from the Parker spiral result.

We should expect, then, as we observe, that the heliospheric field needs to be described on multiple scales. On the largest scale is the Parker spiral, caused by solar rotation. There should also be deviations from the Parker spiral, due to the additional systematic motions of the magnetic field at the Sun and its interplay with variations in the solar wind speed, and also due to the braiding of the field from diffusion at the Sun. These deviations from the Parker spiral occur on a mesoscale. Finally, there should also be small-scale turbulence, driven by Alfven waves, and perhaps by sound waves in the outer heliosphere where the pickup ion pressure dominates. Our challenge is to consider how to describe the interactions among the magnetic fields on these various scales, and thus their evolution in the heliosphere and the consequences.

\section{INTERACTIONS OF THE LOCAL MEAN FIELD AND SMALL-SCALE TURBULENCE}

Consider the interactions between the local mean field - that is, the Parker field plus the mesoscale field, the local, relatively large-scale deviations from the Parker field and the small-scale turbulence. Thus, the mean field $\mathbf{B}$ is

$$
\mathbf{B}=\mathbf{B}_{p}+\Delta \mathbf{B},
$$

where $\mathbf{B}_{p}$ is the Parker field and $\Delta \mathbf{B}$ is the mesoscale field; on the scale of the heliosphere $\langle\Delta \mathbf{B}\rangle$ averages to zero, but locally it can easily be comparable to $\mathbf{B}_{p}$. We also have a mean flow speed, $\mathbf{u}_{s w}$, the solar wind speed, and a turbulent plasma velocity $\delta \mathbf{u}$, which generates fluctuations in the field, $\delta \mathbf{B}$, and visa versa. The field and the plasma are related by an inductance equation,

$$
\frac{\partial \mathbf{B}}{\partial t}=\nabla \times\langle\delta \mathbf{u} \times \delta \mathbf{B}\rangle+\nabla \times\left(\mathbf{u}_{s w} \times \mathbf{B}\right),
$$

where we assume, as usual, that the electric conductivity is infinite. For simplicity, we take the plasma velocity fluctuations to be gyrotropic about the mean field, and not to have components that are correlated with each other. We then look for the consequences of correlations between $\delta \mathbf{u}$ and $\delta \mathbf{B}$.

We can apply a straightforward quasi-linear approach [9] and find that

$$
\frac{\partial \mathbf{B}}{\partial t}=-\nabla \times \nabla \times(\kappa \mathbf{B})+\nabla \times\left(\mathbf{u}_{s w} \times \mathbf{B}\right),
$$

where the effective diffusion coefficient is

$$
\kappa=\frac{1}{2}\left\langle\delta u(t) \cdot \int_{-\infty}^{0} \delta u\left(t-t^{\prime}\right) d t^{\prime}\right\rangle
$$


or, equivalently, $\boldsymbol{\kappa}$ is the correlation between $\delta \mathbf{u}$ and the distance, essentially the mean free path, over which the plasma moves executing $\delta \mathbf{u}$. Equation (3) can also be reduced to a standard diffusion equation by vector identity,

$$
\frac{\partial \mathbf{B}}{\partial t}=\nabla^{2}(\kappa \mathbf{B})+\nabla \times\left(\mathbf{u}_{s w} \times \mathbf{B}\right) .
$$

Thus, if there is a curl in the magnetic field on the mesoscale, perhaps due to braiding of the field, the small-scale turbulence will tend to dissipate the curl by diffusion. We note that the diffusion coefficient is inside the operator in equation (5), unlike the case for energetic particle diffusion. This is the correct form when the diffusion is caused by an external medium [15]. This nuance will not be significant for our subsequent discussions.

Equation (3) is also derived in standard dynamo theory, except here we do not have the so-called alpha effect. First-order smoothing dynamo theory uses the same assumptions and techniques, except the turbulent velocities are assumed to be rotational with a preferred orientation. We assume that the small-scale turbulence is random, without the helical motions required in dynamo theory. We get only diffusion and not a dynamo.

Thus, we argue that there are many processes imparting mesoscale variations into the heliospheric magnetic field, and that small-scale turbulence will tend to dissipate these variations. This is clearly an ongoing process. We impart mesoscale variations at the Sun and they evolve and perhaps grow in the heliosphere. It is also possible that in the outer heliosphere where the pickup ion pressure is important, that variations in that pressure can generate mesoscale variations in the field, and so there should be a continuous generation and dissipation process as the solar wind transports the field outward. We should then ask whether there is any evidence for such a process.

\section{THE LARGE-SCALE ELECTRIC FIELD AND THE ACCELERATION OF ENERGETIC PARTICLES}

If we compare equation (3) with Maxwell's equation,

$$
\frac{\partial \mathbf{B}}{\partial t}=-\frac{1}{c} \nabla \times \mathbf{E}
$$

We note immediately that there is a large-scale electric field,

$$
\mathbf{E}=\frac{\kappa}{c} \nabla \times \mathbf{B}-\frac{\mathbf{u}_{s w}^{\prime}}{c} \times \mathbf{B},
$$

where we have absorbed $\nabla \kappa$ into the mean velocity, $\mathbf{u}_{s w}^{\prime}=-\nabla \kappa$. A gradient in kappa plays the same role as the mean velocity. The electric field is then the sum of the usual motional electric field and the additional term $\kappa \nabla \times \mathbf{B} / c$. The additional term arises 
simply because there is a correlation between the turbulent velocities and perturbations in the magnetic field. In other words, even though on a very local scale we have only the convective electric field, the fact that $\delta \mathbf{u}$ and $\delta \mathbf{B}$ are correlated gives a non-motional, large-scale electric field. The same effect arises in dynamo theory. Because the plasma is actively diffusing, due to the turbulence, it behaves as if it has a finite conductivity.

With an electric field we can accelerate particles. The calculation of the rate of acceleration is straightforward [9]. The energetic particles undergo an $\mathbf{E} \times \mathbf{B}$ drift, with velocity $\mathbf{v}_{E}$.

$$
\mathbf{v}_{E}=\frac{c \mathbf{E} \times \mathbf{B}}{B^{2}}=\frac{\kappa}{B^{2}} \nabla \times \mathbf{B} \times \mathbf{B}+\mathbf{u}_{s w \perp}^{\prime} .
$$

The drift is the usual convective velocity — particles must move with the magnetic field - plus an additional drift due to the extra electric field. The particles are convected at the total drift velocity, $\mathbf{v}_{E}$, and thus the mean rate of energy $T$ change that they experience can readily be shown to be simply

$$
D_{T} \equiv \frac{\Delta T}{\Delta t}=-\frac{2 T}{3} \nabla \cdot \mathbf{v}_{E}
$$

where we have assumed the particles are non-relativistic. Consider the case of a relatively weak acceleration in which the magnetic field does not evolve substantially over distance; i.e., it follows the Parker field in magnitude, as is observed. This can occur if the mesoscale field is continually being regenerated. There must be sufficient energy in, e.g., the pickup ions, to produce the regeneration. The mean acceleration rate can then be shown to be [10]

$$
D_{T}=\frac{2 T \kappa(\nabla \times \mathbf{B})^{2}}{3 B^{2}}-\frac{2 T}{3} \nabla \cdot \mathbf{u}_{s w \perp}^{\prime} .
$$

We should also expect that there is a statistical acceleration, since the mesoscale conditions vary. As was discussed in Fisk [22], the mean and the statistical rate of acceleration must be uniquely related, such that

$$
D_{T T}=\frac{(\Delta T)^{2}}{\Delta t}=\frac{4 T^{2} \kappa(\nabla \times \mathbf{B})^{2}}{3 B^{2}} .
$$

We can use these acceleration rates in a standard cosmic ray transport equation [e.g., 22],

$$
\frac{\partial}{\partial T}\left(D_{T T} \frac{\partial U}{\partial T}\right)-\frac{\partial}{\partial T}\left(D_{T} U\right)-\nabla \cdot\left(\mathbf{u}_{s w}^{\prime} U\right)=0,
$$

where we have assumed that the particles do not undergo significant spatial diffusion. The forms of the acceleration rates in equations (10) and (11) yield power-law spectra as 
solutions to equation (12). Power-law tails on the pickup ion distributions are in fact observed ubiquitously in the solar wind by Gloeckler et al. [1,2]. With reasonable choices for the parameters, the correct spectral index is possible [10]. Thus, the ubiquitous tails on pickup ion distributions may be manifestations of the dissipation of mesoscale variations in the heliospheric magnetic field by the process described here.

\section{THE TERMINATION SHOCK OF THE SOLAR WIND}

There is some controversy at present as to whether the Voyage 1 spacecraft has crossed the termination shock of the solar wind. Krimigis et al. [7] argue that it has, based on observations of accelerated low-energy particles and their absence of convective anisotropies. Burlaga et al. [8] argue that Voyager 1 has not crossed, primarily on the basis of the absence of any measurable increase in the magnetic field strength. Consider then the impact on this argument from the dissipation of mesoscale variations in the magnetic field and the acceleration of energetic particles, as described above.

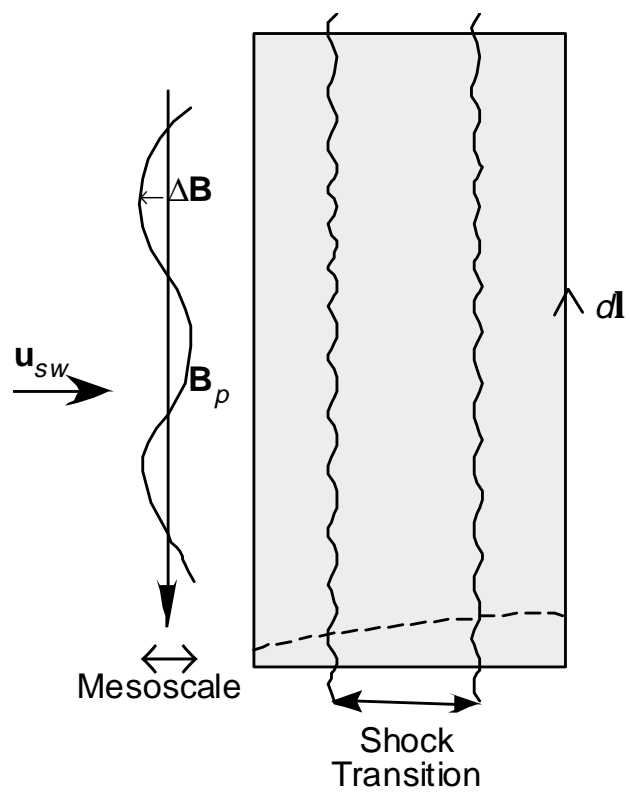

FIGURE 1. An illustration of the termination shock of the solar wind. The shock transition is taken to be thicker than the dimension of the mesoscale variations in the heliospheric magnetic field, which are convected into the shock transition with the solar wind. The shaded area, and the surrounding line integral are used in determining equations (13), (14), and (15).

We have to decide first what the structure of the termination shock is likely to be. We shall consider here, as did Zank [23], that the termination shock is not sharp, but rather 
broader than our mesoscale lengths, and aligned with the Parker field direction essentially parallel to the shock front, as is shown schematically in Fig. 1.

The standard calculation to determine the behavior of the electric field at a shock is to use the steady-state form of equation (6), or $\nabla \times \mathbf{E}=0$; perform a surface integral over the surface shown in Fig. 1; convert it into a line integral; and conclude that along this line,

$$
\oint \mathbf{E} \cdot d \mathbf{l}=0
$$

We have chosen the surface in Fig. 1 to be very much longer parallel to the shock front than perpendicular, in which case the contribution from the short sides of the line integral can be ignored, and we find that the average value (averaged over the segment of the line integral) of the tangential component of the electric field must be continuous, or

$$
\left\langle E_{\text {tangential }}\right\rangle_{\text {upstream }}=\left\langle E_{\text {tangential }}\right\rangle_{\text {downstream }} .
$$

The mesoscale variations average out in performing equation (14), $\langle\Delta \mathbf{B}\rangle=0$, and thus the standard argument simply predicts that the Parker component of the magnetic field should increase inversely as the velocity decreases, the standard frozen-in flow result, or

$$
u_{s w} B_{p}(\text { upstream })=u_{s w} B_{p}(\text { downstream }) .
$$

However, suppose the small-scale turbulence increases in the shock front, sufficient so that there is considerable dissipation of the mesoscale variations. Braided and twisted fields become less so, and the field magnitude will increase much less than we expect, and under certain conditions not at all.

If we are dissipating magnetic field energy, particles should be accelerated. As is discussed in Fisk [10], in the extreme case that the field magnitude doesn't change at all, the dissipation rate should be comparable to the rate at which the field is being compressed. This specifies the magnitude of the acceleration in equations (10) and (11) from which the spectral index of the accelerated particles can be determined through equation (12). We can then demonstrate that the power law indices that are observed by Krimigis et al. [7], are consistent with an increase in the intensity of the accelerated particles by a factor of $\sim 100$, again, as is observed.

Thus, there is a consistency between the fact that the energetic particle intensity increases and the field strength does change if one assumes that the energy put into the field compression is dissipated by the particle acceleration. Does this mean that we crossed the termination shock and Krimigis et al. are right? Not necessarily, it only means that it is possible to cross the termination shock without an apparent change in the magnetic field, and the absence of a field change is consistent with the strong particle acceleration observed. 


\section{CONCLUDING REMARKS}

We have attempted to point out in this paper a relatively simple process, which has potentially far-reaching implications for the heliosphere. Various mechanisms, mainly occurring back at the Sun, will introduce mesoscale variations in the heliospheric magnetic field that result in locally significant curls in the magnetic field, which in turn dissipate by diffusion in small-scale turbulence. This dissipation can result in the acceleration of energetic particles, consistent with the observed superthermal tails on the low-energy energetic particle distributions, and as now seen at or near the termination shock of the solar wind.

\section{ACKNOWLEDGMENTS}

This work was supported, in part, by NSF contract ATM0318590, by NASA contract NAG5-10975, and by JPL contract 1237843.

\section{REFERENCES}

1. G. Gloeckler, L. A. Fisk, T. H. Zurbuchen and N. A. Schwadron, "Sources, Injection and Acceleration of Heliospheric Ion Populations" in Acceleration and Transport of Energetic Particles Observed in the Heliosphere, edited by R. A. Mewaldt et al., AIP Conference Proceedings 528, Melville, New York, 2000, pp. 221-228.

2. G. Gloeckler, "Ubiquitous Suprathermal Tails on the Solar Wind and Pickup Ion Distributions" in Solar Wind Ten, AIP Conference Proceedings 679, 2003, pp. 583-588.

3. L. A. Fisk, J. Geophys. Res. 81, 4633-4640 (1976a).

4. J. A. Le Roux, W. H. Matthaeus and G. P. Zank, Geophys. Res. Lett. 28, 3831-3834 (2001).

5. N. A. Schwadron, L. A. Fisk and G. Gloeckler, Geophys. Res. Lett. 23, 2871-2874 (1996).

6. J. Giacalone, J. R. Jokipii and J. Kota, Astrophys. J. 573, 845-850 (2002).

7. S. M. Krimigis et al., Nature 426, 45-48 (2003).

8. L. F. Burlaga et al., Geophys. Res. Lett. 30, 2072, doi:10.129/2003GLO018291 (2003).

9. L. A. Fisk, Astrophys. J., submitted (2004a).

10.L. A. Fisk, Astrophys. J., submitted (2004b).

11.T. H. Zurbuchen, R. von Steiger, W. B. Manchesterm and L. A. Fisk, "Heliospheric Magnetic Field Configuration at Solar Maximum Conditions" in Proceedings of the Third IGPP Conference, edited by V. Florinski et al., AIP Conference Proceedings (this issue), Melville, New York, 2004, in press.

12.E. N. Parker, Astrophys. J. 128, 664-676 (1958).

13.L. A. Fisk, J. Geophys. Res. 101, 15,547-15,554 (1996).

14.L. A. Fisk, T. H. Zurbuchen and N. A. Schwadron, Space Sci. Rev. 87, $43-54$ (1999).

15.L. A. Fisk, and N. A. Schwadron, Astrophys. J. 560, 425-438 (2001).

16.R. B. Leighton, Astrophys. J. 140, 1547 (1964).

17. Y.-M. Wang and N. R. Sheeley, Astrophys. J. 375, 761-770 (1991).

18. Y.-M. Wang, N. R. Sheeley and J. Lean, Geophys. Res. Lett. 27, 621-624 (2000).

19. C. J. Schrijver, Astrophys. J. 547, 475-490 (2001).

20.C. J. Schrijver and A. M. Title, Astrophys. J. 551, 1099-1106 (2001).

21. U. Feldman, K. G. Widing and H. P. Warren, Astrophys. J. 522, 1133-1147 (1999).

22.L. A. Fisk, J. Geophys. Res. 81, 4641-4645 (1976b).

23. G. P. Zank, "Structure and properties of the heliospheric termination shock" in Proceedings of the Third IGPP Conference, edited by V. Florinski et al., AIP Conference Proceedings (this issue), Melville, New York, 2004, in press. 